\title{
The role of anticholinergic therapy based on the upoint system in the treatment of chronic prostatitis
}

\author{
Kamil Fehmi Narter ${ }^{1}$, Utku Can ${ }^{2}$, Alper Cosskun ${ }^{2}$, Kubilay Sabuncu ${ }^{2}$, Fatih Tarhan ${ }^{2}$ \\ ${ }^{1}$ Acibadem Mehmet Ali Aydinlar University, Urology, Istanbul/Turkey; \\ ${ }^{2}$ Department of Urology, University of Health Sciences, Kartal Dr. Lütfi Kırdar Training and Research Hospital, Istanbul/Turkey.
}

\begin{abstract}
Summary Objective: Chronic prostatitis/chronic pelvic pain syndrome (CP/CPPS) is a common problem and severely impairs the quality of life (QoL). We aimed to investigate the effects of different treatment options on voiding symptoms and QoL in patients with urinary phenotype according to the UPOINT system.

Matherial and methods: Ninety-six patients with NIH category II,III CP/CPPS were included in the study prospectively. After the diagnosis, the questionnaires including NIH Chronic prostatitis Symptom Index (NIH-CPSI), International Prostate Symptom Score (IPSS), Overactive Bladder Screening Questionnaire (OAB-V8), and Beck depression inventory were filled by the patients. The patients with urinary phenotype were treated by alpha-blocker, antimuscarinic or both therapy modalities (combined) considering the specific therapy recommendations by UPOINT. The questionnaires applied on the first visit were reapplied after one month and treatment success was evaluated.

Results: Seventy-three patients were included in 'Urinary phenotype' group (76\%) and 23 were included in 'other phenotypes' (24\%) group of the patients according to the UPOINT classifcation. Significant improvements of symptoms were observed with the all treatment modalities when the NIH-CPSI, IPSS and $O A B-V 8$ scores were compared before and after treatment in the 'Urinary phenotype' group. Significant differences in the percentage of change in values were obtained in the anticholinergic group for pain subdomain of NIH-CPSI and IPSS scores. Conclusion: U-POINT clasification is useful for deciding on the treatment modality in CP/CPSS patients. We showed anticholinergic therapy might be effective option. Addition to the symptomatic recovery, there is need more further studies about effectivity cholinergic system in the prostate tissue.
\end{abstract}

KEY WORDS: Chronic prostatitis; Anticholinergic therapy, UPOINT system.

Submitted 28 August 2018; Accepted 25 September 2018

\section{INTRODUCTION}

Chronic prostatitis/chronic pelvic pain syndrome (CP/CPPS) is a common disease especially seen in men younger than 50 years old. Its prevalence was reported from 2 to $16 \%$ in the male population $(1,2)$. CP/CPPS has a significant negative impact on quality of life and it may cause depresssion and anxiety due to pyschological effects. This syndrome has not been well described yet and its optimal treatment is not clear. Moreover, there is no standard diagnostic test for CP/CPPS. The diagnosis of this problematic disease is only on the basis of symptoms such as pain/discomfort in the pelvic area or lower urinary tract symptoms (LUTS) like storage symptoms frequency and urgency $(3,4)$. Antibiotics, alpha-adrenergic blockers, and anti-inflammatory drugs may be chosen in the treatments for CP/CPSS, but anticholinergic treatment for CP/CPSS has not been preferable yet adequately, and there are few references about this topic (5). According to the National Institutes of Health (NIH), inflammation of the prostate can be classified as acute bacterial prostatis (category I), chronic bacterial prostatitis (category II), chronic prostatitis/chronic pelvic pain syndrome (CP/CPPS, category III) and asymptomatic prostatitis (category IV) (6). CPSS are further subdivided by the presence of inflammation in the extraprostatic secretions or semen (category IIIa) or the absence of it (category IIIb). Although there is no symptoms of disease, chronic prostatitis can be declared histologically on many prostate biopsy reports. The UPOINT system was described in 2008. Patient's symptoms were seperated into six phenotypes as (U)rinary symptoms, (P)sychological dysfunction, (O)rgan specific symptoms, (I)nfectious causes, (N)eurologic dysfunction and $(\mathrm{T})$ enderness of the pelvic floor muscles according to the this system (7). Moreover, comorbidities are often present along with CP/CPSS such as irritable bowel syndrome and fibromyalgia. Recently, a (S)exual dysfunction domain (UPOINT(S)) was described as an additional content to the clinical phenotyping of CP/CPPS (8).

Until today, anticholinergic therapy for patients with CP/CPSS has been very few reported as a symptomatic treatment option for voiding problems.

Our theory are based on cholinergic system effective on the infectious/inflammation process in the prostate tissue. So that, anticholinergic therapy can be a new alternative and additional therapy option for these patients. Many patients with CP/CPSS may have LUTS and genital/pelvic pain. It depend on this, new individualized treatment modalities for patients with CP/CPPS has been considered as a multimodal therapy based on UPOINT sysytem.

For this reason, we aimed to classify patients with $\mathrm{CP} / \mathrm{CPSS}$ according to the UPOINT system and investigate the effects of different treatment modalities such as anticholinergic treatment on voiding symptoms and quality of life in a prospective clinical trial. 


\section{Materials AND Methods}

Ninety-six patients with symptoms of CP/CPPS who were referred to our outpatient clinic between March 2014 and May 2015 were enrolled in this prospective study. All patients were evaluated with a detailed medical history, physical examination, and laboratory tests (urine analysis, two glass test, urinary sonographic evaluation, uroflowmetry, and postvoid residual urine volume-PVR). All patients were also asked to fill out National Institutes of Health Chronic Prostatitis Symptom Index (NIH-CPSI) (9), International Prostate Symptom Score (IPSS) (10), Overactive Bladder Screening Qustionare version 8 (OABV8) (11), and Beck depression inventory (12).

Validated Turkish versions of these all questionnaries are used in the study. NIH categories was designated by the number of leucocytes and culture analysis in the expressed prostate secretion (EPS) examination (modified Meares and Stamey test/two glass test-1968). National Institutes of Health Chronic Prostatitis Symptom Index (NIH-CPSI), International Prostate Symptom Score (IPSS), Overactive Bladder Screening Questionnaire version 8 (OABV8), and Beck depression inventory were used to grade the symptoms. Patients aged 20 to 50 years and patients with CP/CPSS (NIH category II, IIIa and IIIb) with pelvic pain/discomfort for 3 or more months, negative urine culture, maximum urinary flow rate of $15 \mathrm{ml} / \mathrm{sec}$ or greater were included to study. Patients with medical history of pelvic surgery/previous prostate surgery, benign prostate hyperplasia (BPH), urinary obstruction or high postvoid residual volume $(>100 \mathrm{cc}$ ), urinary tract infection, prostatic cancer, urethral stricture, diabetes mellitus, neurogenic lower urinary tract dysfunction and patients who had 5-alpha reductase inhibitors or anticholinergics were excluded from the study.

After the NIH-CPSI, IPSS, OAB-V8 and Beck depression inventory evaluations, patients were clinically classified as 'urinary phenotype' or 'other phenotypes' according to UPOINT system. Alpha blocker (silodosin $8 \mathrm{mg} /$ daily), antimuscarinic (propiverin $30 \mathrm{mg} /$ daily), or combination therapies have been ordered for patients with urinary phenotype, taking into consideration failure of treatments and allergy records in medical history. All patients were classified into the treatment groups as patients with high voiding subdomain of IPSS score were treated with the alpha blocker, patients with high OAB-V8 score were treated with the anticholinergic or patients with both criteries were treated with the alpha blocker and anticholinergic in combined group. Addition to these cut off (IPSS $\geq 8$ and OABV8 $\geq 8$ ) values, for the patients with modest and severe depression, cut off value of Beck Depression Inventory was accepted 17 or higher. NIH-CSPI score was evaluated as a succesful with at least a 6-point improvement and experienced improvements in every domain. When similar questionnaire results were obtained, treatment option were selected according to preference of clinician. One month later, all patients were recalled for control, then all questionnaires were applied again and effectiveness of treatment was evaluated. This study was approved by our Instutional Review Board (03.06.2014/8) and was conducted according to the Declaration of Helsinki. All patients gave informed consent.

Data were presented as median + standard deviation (SD). Stastical analysis was performed by Mann-Whitney
U, Kruskal-Wallis and Wilcoxon tests with SPSS 12.0 (SPSS Inc. Chicago, IL, USA) and $\mathrm{p}<0.05$ was considered to indicate significance. The difference in values before and after treatments was defined as ' $\Delta$ ' and $(\Delta$ /value before treatment) x100 was defined as ' $\%$ change'.

\section{Results}

Based on the U-POINT scoring system, patients were classified as 'urinary phenotype' (n: 73, 76\%) and 'other phenotypes' (n: 23, 24\%). The mean age, duration of symptoms, voiding volume and prostate volume were similar between two groups. LUTS were found to be more frequent in the group of 'urinary phenotype'. Maximum flow rate at voiding in the 'urinary phenotype' group was significant lower than the 'other phenotypes'. NIH-CPSI, IPSS and OAB-V8 scores were statistically significant higher in the 'urinary phenotype' group ( $\mathrm{p}<$ 0.001 ). Stastistically difference was obtained in urinary and QoL subdomain of NIH-CPSI, except pain subdomain between urinary and other phenotypes group. Significant differences were also obtained in IPSS subdomain between 'urinary phenotype' and 'other phenotypes'. But Beck depression inventory scores were similar between two groups (Table 1).

Three patients with positive prostatic secretion culture (Category II) were treated with antibiotics and the remaining 73 patients with non-bacterial prostatitis ( 25 patients with category IIIa, 48 patients with category IIIb) were treated with appropriate medical agents. Moreover, alphablockers (n: 19), anticholinergics (n: 16) and combination therapies (n: 38) were initiated to patients in the "urinary phenotype' group, while psychotherapy or physiotheraphy (n: 6) and food supplements contain quercetin (n: 13) were preferred in the group of 'other phenotypes'. Lifestyle changing and dietery modifications was recommended for all patients (Table 2).

\section{Table 1.}

Evaluation of clinical and demographic data of patients with and without predominant urinary symptoms according to U-POINT score.

\begin{tabular}{|c|c|c|c|}
\hline & & U-POINT & \\
\hline & $\begin{array}{c}\text { Urinary } \\
\text { phenotype }(n=73) \\
\text { median + SD }\end{array}$ & $\begin{array}{c}\text { Other } \\
\text { phenotypes }(n=23) \\
\text { median + SD }\end{array}$ & P* \\
\hline Age & $37+9.6$ & $36.5+9$ & 0.708 \\
\hline Duration of symptoms (months) & $12+47$ & $12+15.5$ & 0.235 \\
\hline $\mathbf{Q}_{\max }$ & $21+8$ & $27+6.3$ & 0.002 \\
\hline Prostate volume (cc) & $22+7.5$ & $20+8.1$ & 0.860 \\
\hline NIH-CPSI & $24+7.3$ & $19+5.8$ & $<0.001$ \\
\hline Pain & $10.5+4.9$ & $10+4.3$ & 0.45 \\
\hline Urinary & $7+2.7$ & $2+1.4$ & $<0.001$ \\
\hline QoL & $8+2.3$ & $6+2$ & 0.002 \\
\hline \multicolumn{4}{|l|}{ IPSS } \\
\hline IPSS & $15.5+8.1$ & $5.5+5.9$ & $<0.001$ \\
\hline QOL & $5+2.3$ & $3+1.3$ & $<0.001$ \\
\hline OAB-V8 & $18+8.4$ & $7+5.6$ & $<0.001$ \\
\hline BECK & $8.5+8.1$ & $9 . .5+7.1$ & 0.968 \\
\hline
\end{tabular}


Table 2.

Treatment chart for patients with and without predominant urinary symptoms according to U-POINT score.

\begin{tabular}{|lcc|}
\hline Treatment & $\begin{array}{c}\text { U-PoINT } \\
\text { Urinary phenotype }(\mathbf{n}=\mathbf{7 3}) \\
\mathbf{n}(\%)\end{array}$ & $\begin{array}{c}\text { Other phenotypes }(\mathbf{n}=\mathbf{2 3}) \\
\mathbf{n}(\%)\end{array}$ \\
\hline Antibiotics & 0 & $3(13)$ \\
\hline Alpha blocker & $19(26)$ & 0 \\
\hline Anticholinergic & $16(22)$ & $1(4)$ \\
\hline Combined & $38(52)$ & 0 \\
\hline Quercetin & 0 & $13(57)$ \\
\hline Others & 0 & $6(26)$ \\
\hline Total & $73(100)$ & $23(100)$ \\
\hline
\end{tabular}

Table 3.

Assessment of pre and post treatment NIH-CSPI, IPSS, OAB-V8 and BECK depression inventory scores according to the treatment groups in 'urinary phenotype' group.

\begin{tabular}{|c|c|c|c|c|}
\hline & Alpha blocker $n=19$ & Antimuscarinic $n=16$ & Combined $n=37$ & $2 p$ \\
\hline \multicolumn{5}{|l|}{ NIH-CSPI } \\
\hline \multicolumn{5}{|l|}{ Total } \\
\hline Pretreatment & $20.9+6.6$ & $27.2+7.5$ & $25.1+7.3$ & \multirow[t]{5}{*}{0.267} \\
\hline Posttreatment & $15.6+5.1$ & $20.1+6.3$ & $20+7.3$ & \\
\hline$\Delta^{*}$ & $-5.3+5$ & $-7.1+5.8$ & $-5.1+6.2$ & \\
\hline \% Change* & $-12.2+11.6$ & $-16.6+13.5$ & $-11.8+14.3$ & \\
\hline $1 \mathrm{p}$ & $<0.001$ & 0.001 & $<0.001$ & \\
\hline \multicolumn{5}{|l|}{ Pain } \\
\hline Pretreatment & $8.4+5.2$ & $11.8+5.2)$ & $9.8+4.5$ & \multirow[t]{5}{*}{$0.031^{* *}$} \\
\hline Posttreatment & $6.3+3.8$ & $8+3.6$ & $8.1+3.4$ & \\
\hline$\Delta^{*}$ & $-2.1+2.2$ & $-3.8+3$ & $-1.8+2.9$ & \\
\hline \% Change* & $-9.8+10.7$ & $-18.1+14.5$ & $-8.5+13.7$ & \\
\hline $1 p$ & 0.002 & 0.001 & $<0.001$ & \\
\hline \multicolumn{5}{|l|}{ Urinary } \\
\hline Pretreatment & $6.2+2.9$ & $7.3+2.8$ & $7.1+2.6$ & \multirow[t]{5}{*}{0.894} \\
\hline Postreatment & $4.6+2.2$ & $5.5+2.6$ & $5.3+2.8$ & \\
\hline$\Delta *$ & $-1.5+2.2$ & $-1.8+2$ & $-1.9+2.2$ & \\
\hline \% Change* & $-15.3+22.2$ & $-17.5+19.8$ & $-18.6+22.1$ & \\
\hline${ }^{1 p}$ & 0.012 & 0.003 & $<0.001$ & \\
\hline \multicolumn{5}{|l|}{ QOL } \\
\hline Pretreatment & $6.4+2.3$ & $8.1+1.5$ & $8.1+2.2$ & \multirow[t]{5}{*}{0.858} \\
\hline Postreatment & $4.7+2$ & $6.6+2.1$ & $6.7+2.6$ & \\
\hline$\Delta^{*}$ & $-1.7+2.2$ & $-1.6+2.6$ & $-1.4+2.6$ & \\
\hline$\%$ Change* & $-14+18$ & $-13+21.9$ & $-11.7+18.8$ & \\
\hline $1 p$ & 0.003 & 0.008 & 0.001 & \\
\hline \multicolumn{5}{|l|}{ IPSS } \\
\hline Pretreatment & $14.8+7.2$ & $16.4+8.7$ & $16.8+8.6$ & \multirow[t]{5}{*}{0.053} \\
\hline Posttreatment & $11.3+6.5$ & $10.5+6.3$ & $13.9+7.7$ & \\
\hline$\Delta^{*}$ & $-3.6+4.2$ & $-5.9+4.9$ & $-2.9+3.8$ & \\
\hline \% Change* & $-10.2+11.9$ & $-17+14$ & $-8.3+10.9$ & \\
\hline $1 p$ & 0.003 & 0.001 & $<0.001$ & \\
\hline \multicolumn{5}{|l|}{ OAB-V8 } \\
\hline Pretreatment & $13.5+8$ & $20+6$ & $19.7+8$ & \multirow[t]{5}{*}{0.190} \\
\hline Postreatment & $9.7+6$ & $14.8+6.2$ & $16.9+7.4$ & \\
\hline$\Delta^{*}$ & $-3.8+3.6$ & $-5.3+4.5$ & $-2.8+4.1$ & \\
\hline \% Change* & $-10.5+10.1$ & $-14.6+12.5$ & $-7.9+11.4$ & \\
\hline${ }^{1} \mathrm{p}$ & 0.001 & 0.001 & $<0.001$ & \\
\hline \multicolumn{5}{|l|}{ BECK } \\
\hline Pretreatment & $9.5+6.4$ & $11+8.8$ & $11.6+8.7$ & \multirow[t]{5}{*}{0.223} \\
\hline Posttreatment & $6.3+4.8$ & $9.4+8.7$ & $8+6.4$ & \\
\hline$\Delta^{*}$ & $-3.2+2.9$ & $-1.6+5.1$ & $-3.7+6.1$ & \\
\hline \% Change* & $-5+4.6$ & $-2.5+8.1$ & $-5.8+9.6$ & \\
\hline $1 \mathrm{p}$ & 0.001 & 0.387 & $<0.001$ & \\
\hline \multicolumn{5}{|l|}{${ }^{1}$ Wilcoxon } \\
\hline \multicolumn{5}{|l|}{${ }^{2}$ Kruskal Wallis } \\
\hline \multicolumn{5}{|c|}{${ }^{*} \Delta:$ The difference in values before and after treatments } \\
\hline
\end{tabular}

The prostate is innervated by rich supply of mixed autonomic postganglionic neurons that arise from the pelvic (inferior hypogastric) and the preganglionic parasympathetic neurons joining the pelvic plexus from the pelvic nerve arising from the sacral spinal cord segment (13). Cholinergic innervation is found in the both stromal and glandular epithelial areas of the human prostate for secretion and contraction (14). The prostate secretes many substances into the seminal plasma that includes PSA (serine protease), zinc, citric acid, magnesium, spermine, prostatic acid phosphatase calcium, and accounts for approximately $15 \%$ of volume of the normal human ejaculate.

Moreover, in vitro contraction of isolated prostate can be inhibited by muscarinic receptor antagonists in the human (15-17). Recently, anticholinergic (antimuscarinic) treatment has become more actual for treatment of male LUTS because such drugs work not only bladder but also on the prostate (18). Muscarinic receptors are intensely represented, especially those belonging to the Ml subtype, on glandular epithelial cells whereas M2 subtype receptors are more represented on the stromal cells. Animal data suggest that muscarinic receptors may be important in the genesis of prostatic secretions (19), smooth muscle contraction of the prostatic capsule $(15,17)$ and prostatic growth $(18,20)$. Cholinergic fibres were found in various regions of the prostate including the anterior capsule, peripheral zone, proximal and distal central zones and their density was more than adrenergic fibers (21). Moreover, muscarinic receptors with binding characteristics of 
the M3 subtype are predominant in the rat ventral prostate (22), and Ml subtype is dense in the rabbit vas deferens (23).

Despite of the only small acute urinary retention risk, muscarinic antagonists may be helpful in men with LUTS as well as overactive bladder (OAB). The expression of muscarinic receptors can be correlated with CP/CPSS. Recently, a possible etiological pathway has been described. According to this mechanism, an unfavorable event as trauma or infection leads to an injury-response of the tissue. Inflammation and upregulation of cytokines may lead to additional organ damage involving nerves, blood vessels, smooth muscles, and the loss of urothelium integrity. As we well know, urothelium is a whole unit especially in the trigonum and prostatic ürethra, and some muscle fibers in detrusor and sphincter region continue in the prostatic area, so that it is a functional and anatomic whole unit. The resulting pain may produce contraction of pelvic smooth and skeletal muscles, finally leading to LUTS, ejaculatory pain or pain in other regions such as back and abdomen. Prolonged pain may sensitize central and peripheral nervous systems and finally cause hyperalgesia and allodynia. For this reason, the primary symptoms of CP/CPPS can be pelvic pain and frequency and few physicians prefer anticholinergics empirically for treatment, and there are only hints of treatment with anticholinergics in some of the guidelines (9).

In our study, anticholinergic therapy improved the pain subdomain score associated with CP/CPSS more than the others $(p=0.031)$. According to this result, anticholinergic therapy is the best succesful option for treatment of pain subdomain of NIH-CPSI.

In some actual studies, muscarinic receptors have also been suggested to be implicated in the control of inflammation, cell growth and proliferation $(24,25)$. The muscarinic receptors are also present in the urethra, but their function have not been clarified adequately. The urethral sphincter tone is predominantly regulated by adrenergic nerves, but muscarinic receptors also modulate the tone (26). Muscarinic receptor mediates contraction of the proximal urethra whilst mediating relaxation of the distal urethra (27). All muscarinic receptor subtypes (M1-5) are located on the urinary system, especially M2 receptors mostly occur in the circular muscle layers, and muscarinic M3 receptors in the longitudinal layer. During inflammation expression of muscarinic M5 receptors is increased, especially in the epithelium and cholinergic induced production of nitric oxide (NO) increase (28). We chose propiverine as an anticholinergic in this study because of it is a competitive antagonist with similar affinity for all muscarinic receptor subtypes (29).

Kim et al. presented their results about efficacy of anticholinergics for CP/CPSS at American Urological Association's (AUA) 2010 Annual Meeting and then confirmed this finding with a prospective study in 2011 $(30,31)$. In that study, ninety six patients with CP/CPPS were randomly assigned in a single-blind fashion and received either ciprofloxacin or ciprofloxacin and solifenacin $(5 \mathrm{mg} / \mathrm{d})$ for 2 months. IPSS, NIH-CPSI, IIEF-5 questionnaires and assessment of QoL were used in that study. According to the results of the study, $67 \%$ of patients had urinary symptoms. Similarly, in our study $76 \%$ of patients showed urinary phenotype. On the other hand, the IPSS assessment appears to be a good indicator follow-up in the management of CP/CPPS especially in many patients with severe LUTS. Statistically significant differences in the total score, the pain and sub-domain scores of NIH-CPSI and total score and storage domain score of IPSS were reported according to Kim's research. Moreover, they reported a statistically non significant increase of the total score of IIEF-5 and no statistically significant difference in residual urine. As a result of the study, the efficacy of anticholinergic treatment in CP/CPPS was demonstrated by the improvements in the NIH-CPSI and IPSS total and storage scores. Similar to the results of that study, the NIHCPSI and IPSS total and storage scores improved significantly in the anticholinergic treatment group for patients with CP/CPSS in our study $(\mathrm{p}=0.053)$.

More than $90 \%$ of cases of CP are not associated with a significant bacteriuria, a condition referred to as chronic pelvic pain syndrome (CPPS) and may not respond to antibiotics or other classical treatment options. Many hypotheses have been suggested for the physiopathology of CP/CPSS including infection, inflammation, autoimmunity, neuromuscular spasm or intraprostatic urinary reflux. CP/CPSS is a syndrome, not a disease and patients may have a wide array of symptoms. For this reason, symptomatic treatment is essential for these patients. Symptom severity should be assessed using the $\mathrm{NIH}$ Chronic prostatitis symptom index (CPSI), which is a validated nine question survey that covers the three domains of pain, urinay symptoms and quality of life (32). The UPOINT system was developed to identify clinical phenotypes according to the symptoms and decide for combined multimodal treatment strategies. The UPOINT system (www.upointmd.com) was validated in several clinical trials (33-35). In this system each category has its own treatment. Use of this treatment strategy is starting to become more widespread and is proving its effectiveness. A strong correlation between the number of positive UPOINT domains and the worse total score of the CPSI measured in patients was shown (36).

Shoskes et al demostrated that a majority (84\%) of patients treated based on the UPOINT phenotype had a clinical improvement of CP/CPSS symptoms measured by an at least a 6-point or greater decrease in NIH-CPSI score $(33,34,37)$. Another study about UPOINT clinical phenotyping reported that $75 \%$ of patients had at least a 6-point improvement in CPSI and experienced improvements in every domain (38). In our study, many patients with CP/CPSS had LUTS and we evaluated to all patients according to UPOINT classification.

In addition to the correlation between the UPOINT and CP/CPSS, sexual dysfunction (ED) was added as a specific domain to create UPOINT(S) (12). In this study, the authors suggested that adding sexual dysfunction to the domain system may be helpful, as a sexual dysfunction is a frequent complaint of patients suffering from CP/CPSS. According to this study, the prevalence of sexual dysfunction is $65 \%$ in these patients.

Multimodality treatment strategies that provides superior outcomes over other treatment strategies for this dis- 
ease and it aims to offer a personalized combination therapy. At least combined therapy may show synergistic effects in the management of CP/CPPS. In our study, NIH-CPSI, IPSS and OAB-V8 scoring values were calculated at statistically significant higher level in the "urinary phenotype' group ( $p<0.001)$. We found statistically significant differences between the two groups in the total score and urinary domain of the NIH-CPSI and the total score and storage symptom score of the IPSS.

As a result of NIH-CPSI, IPSS and OAB-V8's data, we can suggest that CP/CPSS is a complex problem and it can effect bladder, prostate and lower urinary tract functions as a whole system. However to prove the effective of anticholinergics in CP/CPPS decrease of absolute values between two groups should be considered during the study. Our data suggest that anticholinergics are effective in the management of CP/CPSS, especially for the treatment of storage symptoms.

In our study, total and storage scores of NIH-CPSI and IPSS improved significantly in the anticholinergic treatment group for patients with CP/CPSS ( $\mathrm{p}=0.053)$. As we well know, UPOINT system may recommends all treatment options for 'urinary phenotype' according to patient's symptoms and preference of the clinician. According to our results, anticholinergics may be a treatment option for many patients with CP/CPSS who have high IPSS scores with modarate or severe LUTS symptoms. Moreover, this effect of antimuscarinics may be explain by the influence of anticholinergic system on the prostate tissue.

Many treatment options for this disease have been used such as alpha blockers, antibiotic therapy, anti-inflammatory drugs and analgesics, antispasmodics, 5-alpha reductase inhibitors (5-ARI), lifestyle changing, psychotherapy, physiotheraphy, local thermotherapy, neuroleptics and anti-anxiolitics, narcotics, acupuncture, extracorporeal shockwave therapy, myofascial trigger point release, biofeedback, food supplements (quercetin, zinc etc), phytotherapy (bioflavonoids), botulinum toxin A injection or occasionally surgical therapy. There have been few studies of the efficacy of anticholinergics for these patients. At least for a symptomatic relief of complaints, anticholinergic treatment may be tried according to the results of our study. But there is a need for a long term, randomized, controlled study to confirm the efficacy of this treatment.

The limitations of the our study are the lack of a questionnaire to assess the sexual performance of the patients such as IIEF-5 and of an evaluation of long-term treatment outcomes. Furthermore, our study was not a large scale and long term research. So that, more randomized, controlled, long-term and large-scale clinical trials are needed. On the contrary, our study was the first to include Beck depression scale together with UPOINT system in patients with CP/CPSS. Although there was a decrease in Beck score after treatment in patients treated with anticholinergics, the change was not significant $(\mathrm{p}=0.387)$.

This positive but statistically insignificant result can be pioneer for entegration of Beck depression scale and UPOINT system that could be named as UPOINT(D; depression) similarly to UPOINT(S) modification.

\section{Conclusions}

As we well know, CP/CPPS is a common, worrisome problem especially for the young men population. Until today, anticholinergic therapy is not a choice for the treatment of this problem according to classical treatment algorithms, but after the introduction of UPOINT system this option has been considered, especially for patients belong urinary phenotype based on UPOINT system. If patients with CP/CPSS according to subgroup of the NIH categorization have lower urinary symptoms (LUTS) such as urgency, frequency, nocturia, increased postvoid residual urine, dysuria, they have to be evaluated with UPOINT system and they are best candidate for anticholinergic treatment.

In this study, we showed that anticholinergic therapy was an effective and preferable option for these patients. In the near future anticholinergic treatment of patients with CP/CPSS will be accepted and take a place in classical treatment algorithms. In addition to the symptomatic recovery in this disease, we believe that it is possible a physiopathological improvement in the tissue of prostate due to anticholinergic effect, because cholinergic system is well reprsented in the whole prostate tissue. There is need for more randomised prospective clinical trials and histological/molecular researches to evaluate tissue receptors in the prostate.

\section{References}

1. Krieger JN, Lee SW, Jeon J, et al. Epidemiology of prostatitis. Int J Antimicrob Agents. 2008; 31 (Suppl 1):S85-90.

2. Marszalek M, Wehrberger C, Hochreiter W, et al. Symptoms suggestive of chronic pelvic pain syndrome in an urban population: prevalence and associations with lower urinary tract symptoms and erectile function.J Urol. 2007; 177:1815-9.

3. Luzzi GA. Chronic prostatitis and chronic pelvic pain in men: aetiology, diagnosis and management.J Eur Acad Dermatol Venereol. 2002; 16:253-6.

4. Liang CZ, Zhang XJ, Hao ZY, et al. An epidemiological study of patients with chronic prostatitis. BJU Int. 2004; 94:568-70.

5. Fall M, Baranowski AP, Elneil S, et al. European Association of Urology. Eur Urol. 2010; 57:35-48.

6. Krieger JN, Nyberg L Jr, Nickel JC. NIH consensus definition and classification of prostatitis. JAMA. 1999; 282:236-7.

7. Shoskes DA, Nickel JC, Dolinga R, et al. Clinical phenotyping of patients with chronic prostatitis/chronic pelvic pain syndrome and correlation with symptom severity. Urology. 2009; 73:538-42.

8. Magri V, Wagenlehner F, Perletti G, et al. Use of the UPOINT chronic prostatitis/chronic pelvic pain syndrome classification in European patient cohorts: sexual function domain improves correlations. J Urol. 2010; 184:2339-45.

9. Litwin MS, McNaughton-Collins M, Fowler FJ Jr, et al. The National Institutes of Health chronic prostatitis symptom index: development and validation of a new outcome measure. Chronic Prostatitis Collaborative Research Network. J Urol. 1999; 162:369-75.

10. Barry MJ, Fowler FJ Jr, O'Leary MP, et al. The American Urological Association symptom index for benign prostatic hyperplasia. The Measurement Committee of the American Urological Association. J Urol. 1992; 148:1549-57. 
11. Acquadro C, Kopp Z, Coyne KS, et al. Translating overactive bladder questionnaires in 14 languages. Urology. 2006; 67:536-40.

12. Beck AT, Ward CH, Mendelson Met al. An inventory for measuring depression. Arch Gen Psychiatry. 1961; 4: 561-71.

13. Vaalasti A, Hervonen A. Autonomic innervation of the human prostate. Invest Urol. 1980; 17:293-7.

14. Chapple CR, Crowe R, Gilpin SA, et al. The innervation of the human prostate gland-the changes associated with benign enlargement. J Urol. 1991; 146:1637-44.

15. Caine M, Raz S, Zeigler M. Adrenergic and cholinergic receptors in the human prostate, prostatic capsule and bladder neck. Br J Urol. 1975; 47:193-202.

16. Hedlund H, Andersson KE, Larsson B. Alpha-adrenoceptors and muscarinic receptors in the isolated human prostate.J Urol. 1985; 134:1291-8.

17. Gup DI, Shapiro E, Baumann M, et al. Contractile properties of human prostate adenomas and the development of infravesical obstruction. Prostate. 1989; 15:105-14.

18. Ventura S, Pennefather J, Mitchelson F. Cholinergic innervation and function in the prostate gland.Pharmacol Ther. 2002;94:93-112.

19. Bruschini H, Schmidt RA, Tanagho EA. Neurologic control of prostatic secretion in the dog.Invest Urol. 1978; 15:288-90.

20. Witte LP, Chapple CR, de la Rosette JJ, et al. Cholinergic innervation and muscarinic receptors in the human prostate. Eur Urol. 2008; 54:326-34.

21. Crowe R, Chapple CR, Burnstock G. The human prostate gland: a histochemical and immunohistochemical study of neuropeptides, serotonin, dopamine beta-hydroxylase and acetylcholinesterase in autonomic nerves and ganglia.Br J Urol. 1991; 68:53-61.

22. Latifpour J, Gousse A, Yoshida M, et al. Muscarinic receptors in diabetic rat prostate.Biochem Pharmacol. 1991; 42 Suppl:S113-9.

23. Levey AI, Kitt CA, Simonds WF, et al. Identification and localization of muscarinic acetylcholine receptor proteins in brain with subtype-specific antibodies. J Neurosci. 1991; 11:3218-26.

24. Profita M, Giorgi RD, Sala A, et al. Muscarinic receptors, leukotriene B4 production and neutrophilic inflammation in COPD patients.Allergy. 2005; 60:1361-9.

25. Kawashima K, Fujii T. Expression of non-neuronal acetylcholine in lymphocytes and its contribution to the regulation of immune function.Front Biosci. 2004; 9:2063-85.

26. Mattiasson A, Andersson KE, Andersson PO, et al. Nerve-mediated functions in the circular and longitudinal muscle layers of the proximal female rabbit urethra.J Urol. 1990; 143:155-60.

27. Nagahama K, Tsujii T, Morita T, et al. Differences between proximal and distal portions of the male rabbit posterior urethra in the physiological role of muscarinic cholinergic receptors. $\mathrm{Br} J$ Pharmacol. 1998; 124:1175-80.

28. Giglio D, Tobin G. Muscarinic receptor subtypes in the lower urinary tract. Pharmacology. 2009; 83:259-69.

29. Maruyama S, Oki T, Otsuka A, et al. Human muscarinic receptor binding characteristics of antimuscarinic agents to treat overactive bladder. J Urol. 2006; 175: 365-9.

30. Kim HJ, Kyung YS, Woo SH, et al. The efficacy of anticholinergics for chronic prostatitis/chronic pelvic pain syndrome in young and middle aged patients -single-blinded, prospective, multi-center study - AUA Annual Meeting Program Abstracts 796, 2010, Volume 183, Issue 4, Supplement, page e311.
31. Kim DS, Kyung YS, Woo SH, et al. Efficacy of anticholinergics for chronic prostatitis/chronic pelvic pain syndrome in young and middle-aged patients: a single-blinded, prospective, multi-center study.Int Neurourol J. 2011; 15:172-5.

32. Litwin MS, McNaughton-Collins M, Fowler FJ Jr, et al. The National Institutes of Health chronic prostatitis symptom index: development and validation of a new outcome measure. Chronic Prostatitis Collaborative Research Network. J Urol. 1999; 162:369-75.

33. Shoskes DA, Hakim L, Ghoniem G, et al.Long-term results of multimodal therapy for chronic prostatitis/chronic pelvic pain syndrome. J Urol. 2003; 169:1406-10.

34. Magri V, Marras E, Restelli A, et al. Multimodal therapy for category III chronic prostatitis/chronic pelvic pain syndrome in UPOINTS phenotyped patients. Exp Ther Med. 2015; 9:658-666.

35. Polackwich AS, Shoskes DA. Chronic prostatitis/chronic pelvic pain syndrome: a review of evaluation and therapy.Prostate Cancer Prostatic Dis. 2016; 19:132-8.

36. Zhao Z, Zhang J, He J, et al. Clinical utility of the UPOINT phenotype system in Chinese males with chronic prostatitis/chronic pelvic pain syndrome (CP/CPPS): a prospective study. PLoS One. 2013; 8:e52044.

37. Shoskes DA, Nickel JC, Kattan MW. Phenotypically directed multimodal therapy for chronic prostatitis/chronic pelvic pain syndrome: a prospective study using UPOINT. Urology. 2010; 75:1249-53.

38. Guan X, Zhao C, Ou ZY, et al. Use of the UPOINT phenotype system in treating Chinese patients with chronic prostatitis/ chronic pelvic pain syndrome: a prospective study. Asian J Androl. 2015; 17:120-3.

\section{Correspondence}

Fehmi Narter, MD, PhD, Assoc Prof (Corresponding Author)

fehminarter66@gmail.com

Acibadem Mehmet Ali Aydinlar University, Urology

Kiziltoprak Istasyon cad. Murat apt.

24/15 Kadikoy 34724 Istanbul (Turkey)

Utku Can, MD

utkucan99@yahoo.com

Alper Coşkun, MD

dr.alper05@gmail.com

Kubilay Sabuncu, MD

kubilaysabuncu@yahoo.com

Fatih Tarhan, MD, Assoc Prof

tarhanf@yahoo.com

Department of Urology, University of Health Sciences, Kartal Dr. Lütfi Kurdar Training and Research Hospital, Istanbul (Turkey) 